Recent Insights into the Physics of the Sun and Heliosphere:

Highlights from SOHO and Other Space Missions

IAU Symposium, Vol. 203, 2001

P. Brekke, B. Fleck, and J. B. Gurman eds.

\title{
Observation of Reconnection Inflow of a Solar Flare
}

T. Yokoyama

National Astronomical Observatory of Japan, Nobeyama Radio

Observatory, Minamimaki, Minamisaku, 384-1305, Japan

K. Akita

Osaka Gakuin Univ., Suita, Osaka, 564-8511, Japan

T. Morimoto, K. Inoue

Kyoto Univ., Kyoto, 606-8502, Japan

J. Newmark

Emergent Information Technologies-East, GSFC/NASA, Greenbelt, MD20771, USA

\begin{abstract}
We find an important piece of evidence for magnetic reconnection inflow in a flare on March 18, 1999. The flare occurred on the north-east limb, displaying a nice cusp-shaped soft X-ray loop and a plasmoid ejection typical for the long-duration-events. As the plasmoid is ejected, magnetic reconnection occurs at the disconnecting point. A clear ingoing pattern toward the magnetic X-point is seen. The velocity of this apparent motion is about $5 \mathrm{~km} \mathrm{sec}^{-1}$, which is an upper limit on reconnection inflow speed. Based on this observation, we derive the reconnection rate as $M_{A}=0.001-0.03$, where $M_{A}$ is a Alfvén Mach number of the inflow.
\end{abstract}

\section{Introduction}

Solar flares are now thought to be caused by magnetic reconnection (e.g. Shibata 1996; Yokoyama \& Shibata 1998). In this model, the antiparallel field lines dissipate in a certain local point in the corona. The tension force of the reconnected field lines then accelerates the plasma out of the dissipation point. In response to this outflow, the ambient plasma is drawn in. The inflowing plasma carries the ambient magnetic field lines into the dissipating point. These field lines continue the reconnection cycle. In this manner the magnetic energy stored near the neutral point is released to become the thermal and bulk-flow energy of plasma. The study shown in this paper is the first successful result of the searching effort of the reconnection inflow (Yokoyama et al. 2001). 

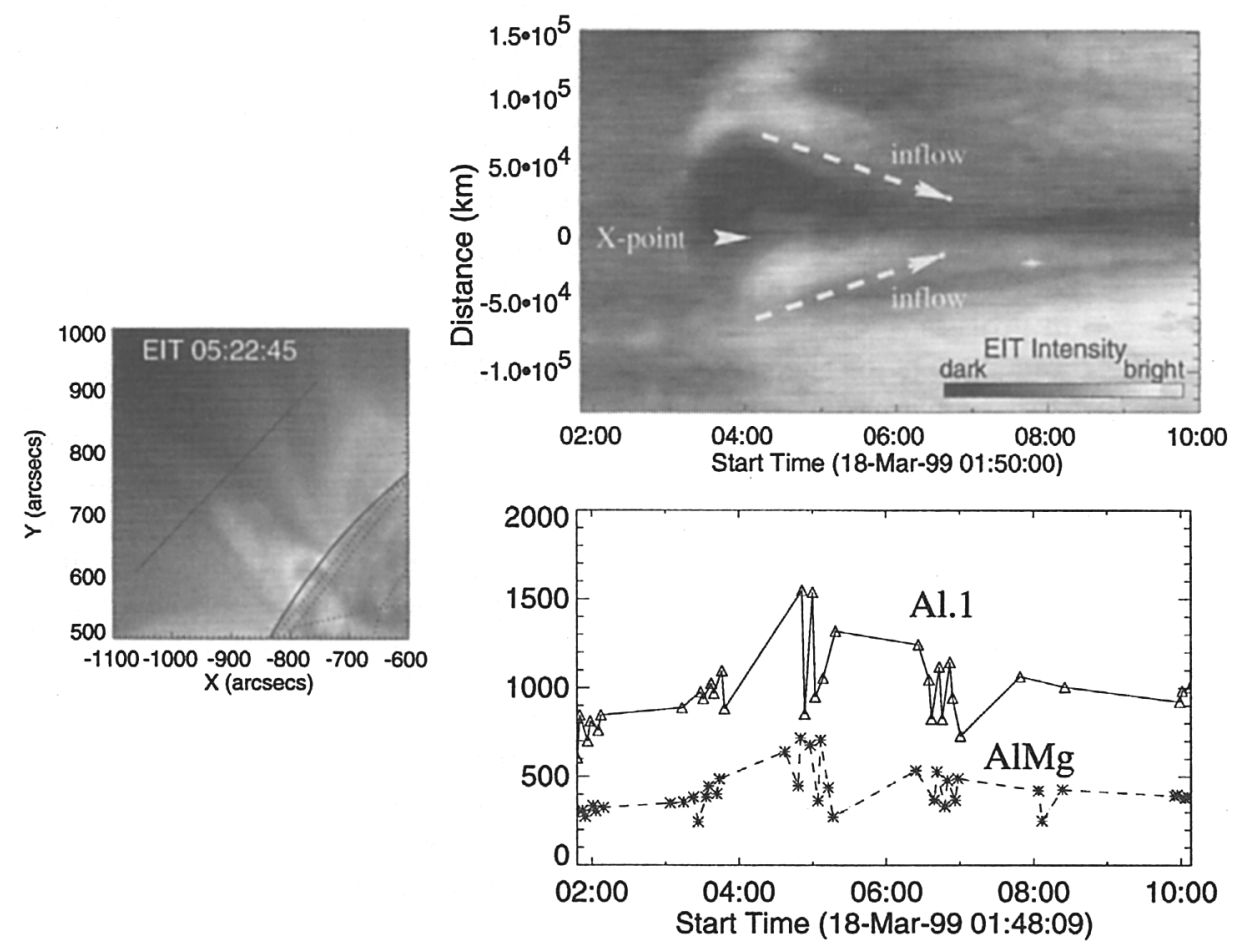

Figure 1. Evolution of 1-dimensional plot of EIT data nearly across the X-point. (left) A snap shot of EUV (Fe $195 \AA$ ) image by $\mathrm{SOHO} / \mathrm{EIT}$. The field-of-view size of this panel is $670 \operatorname{arcsec} \times 670 \operatorname{arcsec}$ $(\approx 350,000 \mathrm{~km} \times 350,000 \mathrm{~km}$ ). (right upper) time evolution of 1 dimensional distribution of EUV intensity along the thick solid line in the upper panel. (right bottom) Soft X-ray light curves by SXT in arbitrary units.

\section{Flare 1999-3-18; Discovery of Reconnection Inflow}

This flare occurred on the north-east solar limb. According to the Yohkoh/SXT soft X-ray observation, it showed a nice cusp-shaped loop and a plasma blob (plasmoid) ejection. The EUV observation of the same flare by SOHO/EIT shows us a bubble-like void ejection. The core of this EUV void corresponds to the soft X-ray plasmoid and is bright in X-ray but dark in EUV because of its temperature $\approx 4 \mathrm{MK}$. As this void moves away from the limb, the lower part of the void becomes thinner and thinner. When the void finally detaches from the limb, an ' $\mathrm{X}$ ' shape structure appears at the detaching point. We believe a magnetic reconnection process occurs at this detaching point because of the following reasons: First, it is natural to consider that the void is a bubble of plasma surrounded by magnetic field lines. As the void detaches, the surrounding lines are also pulled up and at the detaching point the antiparallel field lines meet. Second, as the void continues rising, the pattern of threads (which we 
assume that the threads are field lines) merge toward the ' $\mathrm{X}$ ' point. Figure 1 shows the evolution of the slice cut across the 'X'-point. A clear merging pattern toward the ' $\mathrm{X}$ ' line can be seen. We believe this is the inflow of the magnetic reconnection. Third, as the consequence of this process, reconnected field lines should shrink down below the ' $\mathrm{X}$ ' point. We observe an evolving cusp-shaped loop on the limb in soft X-ray and EUV which is the result of the piling up of the shrunk magnetic field lines. Through these observations, we conclude that we have finally found the last link demonstrating the reality of ongoing magnetic reconnection process.

The measurement of the inflow velocity is done by tracing the movement of the thread-like patterns in the EIT movie. We measure the speed of the movement of a pattern in a slice cut indicated in the left panel of Figure 1. The speed of the incoming pattern is about $V_{\text {pattern }}=4.7 \mathrm{~km} \mathrm{sec}^{-1}$ over the period 4:00-6:00 UT (right upper panel of Fig 1). This measured speed $V_{\text {pattern }}$ is an upper limit of the real inflow speed because it is a mixture of the real inflow velocity $V_{\text {inflow }}$ and an error velocity due to the movement of the X-point. Using simple geometrical consideration one can show that $V_{\text {pattern }}=V_{\text {inflow }}+V_{\mathrm{xp}} \tan \theta$, where $V_{\mathrm{xp}}$ is a velocity of the X-point in the fixed frame and $\theta$ is the angle between the measured thread-like pattern in the images and the perpendicular direction of the slice cut. It is still difficult to measure the values of $V_{\mathrm{xp}}$ and $\theta$ from the images. But from the measurement of the outer edge of the ejecting plasmoid, it is possible to obtain the upper limit values to these variables. Thus, we have $\tan \theta<\tan \theta_{\text {plasmoid }} \approx 0.1$ and $V_{\mathrm{xp}}<V_{\text {plasmoid }} \approx 37 \mathrm{~km} \mathrm{sec}^{-1}$, where $\theta_{\text {plasmoid }}$ is the angle of the outer edge of the plasmoid and $V_{\text {plasmoid }}$ is the velocity of the plasmoid. Then, the derived lower limit of the inflow velocity is $V_{\text {inflow }}>1.0 \mathrm{~km} \mathrm{sec}^{-1}$.

The magnetic reconnection rate, $M_{\mathrm{A}}$ defined as the ratio of the inflow speed to the Alfvén velocity is also obtained. Readers would be reffered to Yokoyama et al. (2001) for the derivation of the Alfvén velocity. Under an assumption that the observed thermal energy increasing rate balances with the Poynting energy flux, the Alfvén speed becomes $C_{\mathrm{A}}=160-970 \mathrm{~km} \mathrm{sec}^{-1}$. Since the inflow speed is $V_{\text {inflow }}=1.0-4.7 \mathrm{~km} \mathrm{sec}^{-1}$, the reconnection rate is $V_{\text {inflow }} / C_{\mathrm{A}}=0.001-$ 0.03 . This observed result gives us further information about the magnetic reconnection process itself. The value of reconnection rate $M_{\mathrm{A}}=0.001-0.03$ is roughly consistent with the Petschek's (1964) reconnection model.

\section{References}

Petschek, H. E. 1964, in Proc. of AAS-NASA Symp. on the Physics of Solar Flares, ed. W. N. Hess, (NASA SP-50), 425

Shibata, K. 1996, Adv. Space Res., 17, 9

Yokoyama, T. \& Shibata, K. 1998, ApJ, 494, L113

Yokoyama, T., Akita, K., Morimoto, T., Inoue, K. \& Nermark, J. 2001, ApJ, 548 , in press 